\title{
Severe Acute Respiratory Syndrome Coronavirus 2 (SARS- Cov-2) and the COVID-19 Pandemic: The Early Months
}

\author{
Terri Rebmann, PhD, RN, CIC, FAPIC ${ }^{1 *}$, Angela Vassallo, MPH, MS, CIC, FAPIC ${ }^{2}$ and Rebecca \\ Taylor Alvino, RN, MS, CNS, CIC, CNOR
}

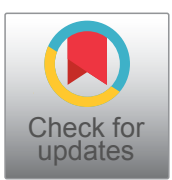

${ }^{1}$ Department of Epidemiology \& Biostatistics, Saint Louis University, College for Public Health and Social Justice, Saint Louis, Missouri, USA

${ }^{2}$ Assistant Professor, West Coast University, Los Angeles, CA, USA

${ }^{3}$ Infection Preventionist, UCSF Health, San Francisco, California, USA

\section{Introduction}

SARS-CoV-2, a novel Coronavirus virus that causes COVID-19 disease, was first identified in China in December, 2019 [1]. SARS-CoV-2 spread globally very rapidly, with the first case appearing in Seattle, Washington in January, 2020 [2]. COVID-19 spread rapidly within the US after that, and was officially declared a pandemic on March 11, 2020. Both healthcare and public health systems were quickly overwhelmed, despite implementing crisis standards of care, social distancing measures, and other health and public health interventions. As of this writing, the COVID-19 pandemic is ongoing and the US tops the world in cases and deaths [3]. The purpose of this paper is to describe the public health aspects of the SARS-CoV-2 pandemic, including a history of novel Corona viruses, the epidemiology of SARS-CoV-2 and how it evolved into a pandemic, the public health and healthcare response in the US during the first few months of the pandemic, public health interventions to flatten the curve and minimize morbidity and mortality, and discuss how the remainder of the pandemic may play out. A timeline of the early events that occurred prior to COVID-19 being declared a pandemic are outlined in Figure 1.

\section{History of Corona Viruses and Novel Corona Viruses}

Corona viruses are RNA viruses that belong to the subfamily Corona viruses. Corona viruses are zoonotic and can infect humans as well as pigs, cattle, horses, rodents, birds, bats, and other animals [4]. Most Corona viruses circulate annually and cause only mild disease similar to the common cold $[4,5]$. However, periodically, a human novel Coronavirus strain emerges. These novel strains have been associated with higher mortality compared to the human Corona viruses that circulate annually [5]. Severe acute respiratory syndrome Coronavirus 2 (SARS-CoV-2) is the third novel Coronavirus to emerge, following SARS-CoV-1 in 2002 and Middle East respiratory Coronavirus (MERS CoV) in 2012. The mortality rates for SARS-CoV-1 and MERS CoV have been approximately $9 \%$ and $36 \%$, respectively [6]. Though outbreaks of both SARSCoV-1 and MERS CoV have occurred, neither has yet developed into a pandemic.

\section{History and Epidemiology of SARS-Cov-2, the Virus That Causes the Disease COVID-19}

SARS-CoV-2, the virus that causes the disease "coronavirus disease 2019" (abbreviated to COVID-19) was first identified in December, 2019 in Wuhan, China. Initial cases were believed to be associated with a seafood market in China and more specifically with pangolins $[4,7]$. However, a genomic analysis of the virus found that it is likely a recombinant virus stemming from a bat Coronavirus and a pangolin or origin unknown Coronavirus [7]. Furthermore, it is likely that the virus was circulating in China as early as November, 2019 [8]. It was originally thought that COVID-19 could only spread to humans through contact with animals, but healthcare personnel providing care to infected patients soon became ill, confirming that human-to-human transmission was occurring [9].

SARS-CoV-2 is a highly contagious disease that spreads rapidly. Epidemiologists use the term $R_{0}$ to describe the infectiousness of a disease, with $R_{0}$ being equal to the average number of secondary cases expected from each primary case. The $R_{0}$ for SARS-CoV-2 is estimated to be 2.2-3.1, which explains the rapid global spread of the disease [10]. Fur-

*Corresponding author: Dr. Terri Rebmann, PhD, RN, ClC, FAPIC, Department of Epidemiology and Biostatistics, Saint Louis University, College for Public Health and Social Justice 3545 Lafayette Avenue Room 463, Saint Louis, Missouri 63104, USA, Tel: (314)-977-8260 Fax: (314)-977-3234

Accepted: December 07, 2020

Published online: December 09, 2020

Citation: Rebmann T, Vassallo A, Alvino RT (2020) Severe Acute Respiratory Syndrome Coronavirus 2 (SARS-Cov-2) and the COVID-19 Pandemic: The Early Months. J Nurs Pract 3(1):212218 


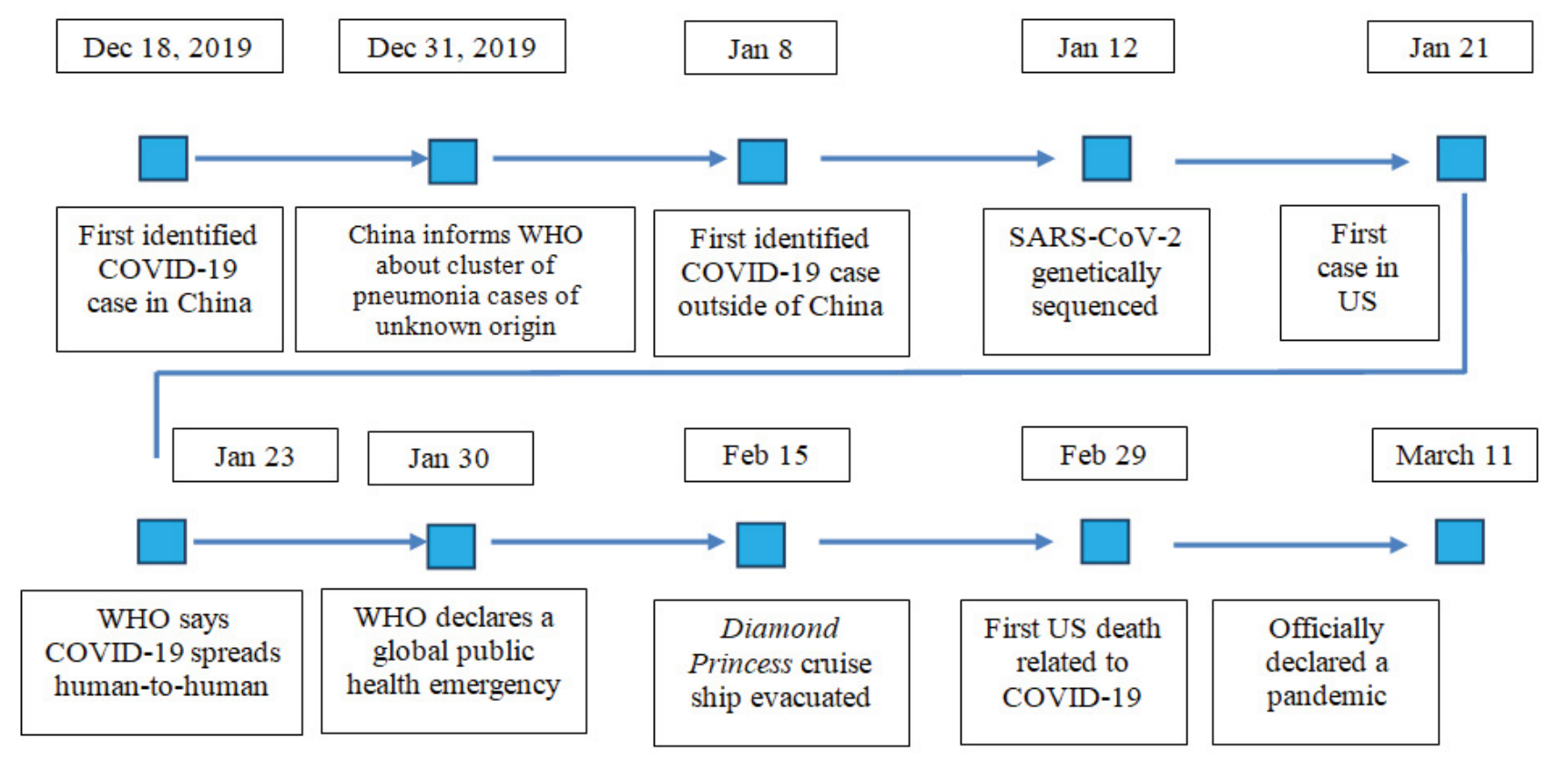

Figure 1: Timeline of events in early part of the COVID-19 pandemic

thermore, asymptomatic or presymptomatic viral shedding has been known to occur, which means that individuals can spread disease before they have symptoms [11]. This makes it difficult to identify infected individuals who require isolation and can lead to further transmission [11]. Case identification has been found to be even more difficult in long term care facilities among residents with cognitive impairment, which has resulted in multiple outbreaks among this vulnerable population [12].

Following the early cases in Wuhan, China in December, 2019 , SARS-CoV-2 spread globally very rapidly. By the end of January, 2020, there were almost 7,700 cases of COVID-19 in China and it had spread to 19 countries, including the United States [13]. On March 11, 2020, the World Health Organization declared COVID-19 to be a pandemic. By April, 2020, almost all countries were experiencing cases or clusters of COVID-19 and more than 40 countries were experiencing community-wide transmission [14].

The early cases of COVID-19 presented as an acute respiratory distress syndrome and was believed to be associated with a viral pneumonia. About half of the original cases had an underlying illness, such as hypertension, cardiovascular disease, or diabetes [13]. The majority of infected individuals ( 80\%) will experience a mild disease course and will not require hospitalization [15]. About 14\% experience severe disease requiring hospitalization, and approximately $5 \%$ will have critical disease requiring intensive care [15]. Before asymptomatic and screening testing were available, the mortality rate appeared high ( $>4 \%$ ) due to the tendency to prioritize hospitalized patients for testing $[15,16]$. In addition, in areas or countries that lacked healthcare surge capacity to accommodate the patient surge, the case fatality rate was found to be high [16]. The overall mortality rate has been estimated to be between 0.25 - 3\%, with higher rates expected in low income countries and geographical regions that cannot accommodate the patient surge [16]. In general, children have a much milder form of disease compared to adults, with fewer than $1 \%$ requiring hospitalization $[15,17]$. Infants under the age of one year are at significantly higher risk of hospitalization and death from COVID-19 compared to children of all other ages [17]. In rare situations, pediatric COVID-19 patients may develop multisystem inflammatory syndrome in children (MIS-C), though epidemiological data is still lacking about whether this is uniquely related to SARSCoV-2 or a clinical inflammatory syndrome that occurs after exposure to other viruses [18]. Those over the age of 65 years are at significantly higher risk of having severe or critical disease and death compared to those of all other ages; mortality rates among the elderly have been found to be between 8-15\% [15]. Individuals with co-morbidities, such cardiovascular disease, diabetes, chronic lung disease, hypertension, and cancer are also at higher risk of hospitalization and death compared to those without underlying illnesses [15].

Multiple localized outbreaks of COVID-19 have occurred across the US that have been associated with vulnerable individuals living in congregate and crowded settings. Examples include long term care facilities, skilled nursing facilities, prisons, and homeless shelters [19]. These settings house highrisk individuals living closely together and often lack the resources needed to implement extensive infection prevention protocols or respond to outbreaks. These smaller outbreaks have contributed to regional illness rates and healthcare surge capacity, as well as overall mortality rates for the community.

\section{Public Health Response within the United States}

Soon after it was discovered that COVID-19 involved human to human transmission, countries outside of China began implementing screening and surveillance to identify po- 
tentially contagious individuals. The hope was to prevent importation of cases by rapidly identifying a contagious individual so that they could be placed into isolation and prevent a localized outbreak. Prior to cases in the U.S., airport screening was used to help identify potentially infected individuals ${ }^{13}$. In addition to airport screening, the U.S. quarantined planes coming from Wuhan, China and multiple cruise ships of repatriated citizens [20]. Travel restrictions were implemented for non-US citizens coming from China and other areas where there was sustained COVID-19 transmission, and the Centers for Disease Control and Prevention (CDC) recommended avoiding nonessential travel [19]. All of these measures were put into place to prevent a COVID-19 outbreak in the U.S. and limit disease spread among individuals on the quarantined flights and cruises [20]. Despite these measures, cases and clusters of COVID-19 were identified in the US, many of which were tied to cruises (prior to the temporary halting of cruise travel), public gatherings, and social events, such as Mardi Gras in Louisiana, a conference in Boston, and a large funeral in Georgia [19]. The first known case of COVID-19 in the US occurred in Seattle, Washington on January 21, 2020 due to a traveler coming from Wuhan, China [2]. COVID-19 spread quickly in the Seattle area, resulting in the highest case counts in the US at that time. In addition, Seattle experienced the first COVID-19 outbreak in a long-term facility, resulting in 129 cases of illness among residents, staff, and visitors, and a $27 \%$ mortality rate among residents [21]. Seattle remained the epicenter of the pandemic in the US until mid-March when New York surpassed Seattle's number of cases.

In mid-March, additional community-based interventions were implemented to try to slow disease spread. Citizens were instructed to isolate or quarantine at home (depending on whether they were infected or believed to have been exposed), large gatherings were restricted or cancelled, and businesses were instructed to transition to telework whenever possible [19]. However, interventions were not implemented uniformly across the US nor even within a single state. For regions with evidence of sustained disease transmission, even more extensive interventions were implemented, including school closures, stay-at-home orders, recommendations to wear a cloth mask when in public, and other social distancing measures such as keeping six feet from others whenever possible [19]. In addition, public health instituted more widespread testing and contact tracing to help identify infected individuals who needed isolation and their contacts who required monitoring and home quarantine. Despite these measures, the US experienced a rapid increase in cases, particularly in urban regions such as Seattle and New York City. This was in part due to the role of asymptomatic disease spread [22], but also just the nature of highly contagious diseases such as COVID-19 in densely populated areas when social distancing measures are not in place.

The public health and social distancing measures implemented in the US were intended to flatten the curve, meaning to lessen the peak and spread out the number of COVID-19 cases over time. These reduced morbidity rates allow healthcare systems to manage the patient surge, which results in lower mortality rates [19]. When healthcare surge capacity is exceeded, mortality rates can increase by $1-2 \%$ [16]. In order to sustain a flattened curve, public health may need to use social distancing measures for extended periods of time or implement them intermittently when increased cases occur in the community [6]. Loosening social distancing restrictions too soon can cause an increase in cases, which could overwhelm healthcare and lead to higher mortality rates [23]. More restrictive social distancing policies have been found to result in major reductions ( 65\%) in COVID-19 cases over a two-week time period [24]. In addition, social events that have occurred without the use of social distancing measures have been found to result in outbreaks involving high attack rates, such as a wedding that infected about $22 \%$ of attendees [25]. Moving forward, the most critical public health interventions will include development of a COVID-19 vaccine, rapid identification of cases through surveillance and testing, contact tracing, and extended or intermittent social distancing measures.

In addition to implementing community mitigation measures such as social distancing, public health has directed resources aimed at preventing and controlling localized outbreaks of COVID-19 within long term care and skilled nursing facilities, prisons, and homeless shelters [19]. Interventions implemented at controlling COVID-19 in these settings include restricting visitors, cohorting patients and staff, rapid case identification, increased cleaning and disinfection, and use of personal protective equipment $[19,22]$. These settings and their clients should also be prioritized for testing to ensure that infected individuals are identified rapidly so they can be isolated [26].

\section{Healthcare Response}

U.S. hospitals and healthcare systems began preparing to identify and respond to COVID-19 in early winter, 2019/2020 as news spread about the threat of SARS-CoV-2 in China and beyond. Active surveillance was implemented to quickly identify infected individuals so they could be placed on isolation. Hospitals began examining their surge capacity plans in anticipation of patient influxes and communities started discussing alternate care sites or other approaches to increasing surge capacity. Areas where imported cases were first identified, including New York City and Los Angeles, began to see an increase in patients, many of whom were suspected of having COVID-19. Many hospitals implemented reverse triage protocols consisting of discharging low acuity patients in order to free up available beds for COVID-19 patients [27]. In late March, 2020, the U.S. Navy deployed its two medical ships, the United States Naval Ship (USNS) Mercy and the USNS Comfort to LA and NYC, respectively, in response to this increased demand to aid in healthcare surge capacity. Both ships were deployed to treat patients who did not have COVID-19 in order to open hospital beds for COVID-19 patients. Cities such as Los Angeles and Phoenix reopened hospitals that had previously closed to use as surge hospitals to care for COVID-19 patients. The state of California leased the closed St. Vincent's Medical Center in downtown LA in a partnership between Los Angeles County Department of Public Health with Dignity and Kaiser health systems co-managing the facility. The Los Angeles Surge Hospital, or LASH, began 
accepting COVID-19 patients on April 13, 2020 with a maximum bed capacity of 266 and an expanded ICU capacity as a transfer-only facility with no emergency department.

The U.S. healthcare system became quickly overwhelmed in some areas, illustrating a lack of capacity in surge planning and supplies. Hospitals and nursing homes became overwhelmed by the high volume of patients, particularly in New York City, Westchester, and Brooklyn [28]. In addition, many U.S. hospitals reported a lack of personal protective equipment (PPE), hand sanitizer, cleaning supplies, ventilators, and medical equipment to sustain the surge $[28,29]$. The lack of general surge capacity, intensive care unit beds, ventilators, and PPE all led to increased mortality rates in the U.S., a phenomena that was also seen in other countries whose healthcare systems could not handle the large patient surge [16].

\section{Supplies from Local, Regional, and Federal Stockpiles}

A key component of mass casualty planning is the stockpiling of supplies and medication at the local, regional, and federal levels [30,31]. Planning organizations and researchers recommend that hospitals, healthcare systems, and regions develop stockpiles of pharmaceutical products, medical supplies, and equipment [31]. In addition, at the federal level, the Strategic National Stockpile (SNS) is available to help supplement jurisdictions responding to public health emergencies. The SNS was first established in 1999 under the name the National Pharmaceutical Stockpile, and consisted only of pharmaceutical products, such as antibiotics and vaccines, designed to help respond to a bioterrorism attack. However, the SNS has evolved over time to include medical supplies and equipment, including ventilators and PPE, to meet changing threats. The sole purpose of the SNS is to ensure that adequate medications and medical supplies can be quickly deployed anywhere within the US to fill gaps in current capacity due to a public health threat such as COVID-19. However, the SNS is not sufficient to aid all jurisdictions in long-term response during a pandemic; it is intended to be supplemental only. As with the 2009 H1N1 pandemic, local, regional, and federal stockpiles was quickly depleted during the COVID-19 pandemic. By April 9, 2020, long before the peak of patient cases, the SNS reported that it had deployed more than $90 \%$ of its PPE supplies [32]. In addition, many criticized the federal government's management and deployment of SNS supplies as being unfair [33].

\section{Healthcare Worker Infection and Fear}

Soon after the first cases of COVID-19 were found in the U.S., cases of occupationally-acquired COVID-19 were identified among healthcare staff, including nurses. For example, at one Californian hospital, 121 healthcare workers were exposed to an asymptomatic COVID-19 patient, 43 of whom became symptomatic and 3 of whom tested positive for SARSCoV-2 [34]. The three healthcare workers who tested positive had not been wearing PPE when providing care to the patient, including during routine physical exams and while performing nebulizer treatments. The healthcare workers who became infected with COVID-19 had spent more time with the pa- tient than those who did not become infected [34]. Healthcare workers have been found to be at risk from occupational exposure and disease when providing care to COVID-19 patients, especially when they do not wear or have access to the appropriate PPE [1]. As PPE supplies diminished in healthcare facilities across the US, \#Get MePPE became a trending hash tag on social media with healthcare workers mounting a rallying cry of fear and frustration for their own safety and that of their patients. The CDC responded by implementing crisis standards of care related to PPE, such as guidance on best practice approaches to the re-use and decontamination of N95 respirators, masks, and other types of PPE [35]. Crisis standards of care are protocols implemented during public health emergencies when standard medical and nursing practices are unable to be met due to patient surges and a lack of equipment.

Thus far in the pandemic, skilled nursing facilities have been especially overwhelmed by increasing COVID-19 patients while simultaneously experiencing dwindling supplies of PPE. Many healthcare workers went on strike in an effort to prevent themselves from acquiring COVID-19 while caring for their patients. The Magnolia Rehab and Nursing Center, a 90 bed skilled nursing facility in Riverside, CA, had to evacuate 84 residents on April 8, 2020 because of employee walk outs due to fear of safe conditions [36]. While 13 certified nursing assistants (CNAs) were scheduled to work on April 8, 2020 only one showed up to work while there were 34 COVID-19-infected patients and 5 COVID-19-infected staff [36]. Patients were transported to a federal medical station established at the Indio Fairgrounds, as well as other healthcare facilities in the area while Bruce Barton, the director of the County's Emergency Management Department made a public plea to healthcare workers for assistance promising to provide payment, malpractice insurance, full PPE, and clean facilities to whomever would show up to provide patient care [36].

Studies have found that outbreaks of emerging infectious diseases and pandemics, including COVID-19, are associated with increased rates of depression, anxiety, fear, and insomnia among healthcare workers [37-39]. Healthcare workers have also reported symptoms of post-traumatic stress disorder and substance abuse when responding to pandemics, particularly when they need to be quarantined or the risk of personal infection is high $[38,40]$. The risk of psychological negative outcomes have been found to be higher when healthcare personnel responding to pandemics lack access to PPE or when they do not feel knowledgeable about how to respond to the disease involved in the pandemic $[38,39,41]$.

\section{Pharmacological Interventions for COVID-19}

At the time of this writing, no efficacious FDA-approved pharmacological intervention exists, such as an antiviral therapy, prophylaxis, or vaccine, though multiple antiviral therapies are being investigated. Both antiviral therapy and vaccine could aid in COVID-19 response and help reduce morbidity and mortality, but a vaccine would be the best intervention to stop the pandemic [42]. However, it has been estimated that the soonest a COVID-19 vaccine could be developed, tested, 
and approved for release is $12-18$ months after identification of the virus, meaning spring or fall 2021 [43]. Furthermore, it will take additional time after a vaccine has been developed and approved for release to have produced sufficient vaccine for the nation. Therefore, as with previous pandemics, there will likely be a prioritization plan developed to outline which groups will receive vaccine first. Prioritization plans for pandemic vaccine are decided based on the event's epidemiology, but always include healthcare personnel and other first responders as a top priority group [42]. There will also be a need for the rapid dispensing of vaccine once one has been developed. This may include the use of open and closed points of dispensing (PODs) for mass vaccination efforts [44]. Hospitals function as closed PODs, so acute care nurses should be able to receive COVID-19 vaccine on-site once the vaccine is available. Many other healthcare settings, such as long-term care facilities and outpatient surgery centers often serve as closed PODs, so nurses working in those settings should also have rapid access to vaccine. For nurses who work in agencies that are not closed POD sites, vaccine will be made available through open POD sites that are available to citizens in all jurisdictions that plan to use PODs for mass vaccination $[44,45]$. Due to the current lack of pharmacological interventions, non-pharmacological interventions (NPIs), such as surveillance, isolation, contract tracing, and social distancing are the most effective ways to control COVID-19 at this time.

\section{Past Influenza Pandemics and Predicting the Remainder of the COVID-19 Pandemic}

It is very challenging to predict how the remainder of the COVID-19 pandemic will unfold. Although researchers have studied past pandemics, all have involved influenza. This is the first known pandemic with a novel Coronavirus and this event may or may not reflect the epidemiology of past influenza pandemics. However, it is useful to examine past influenza pandemics and other human Coronavirus to understand how these events often unfold over time. Four influenza pandemics have occurred during the $20^{\text {th }}$ Century. Past influenza pandemics have often involved waves or surges of infected individuals, with each wave lasting 6-8 weeks [46]. Furthermore, most past pandemics have involved multiple waves, with a first wave occurring the spring and a second larger wave emerging the following fall [42]. It is too soon to tell whether COVID-19 will follow the same pattern as past influenza pandemics, but Coronaviruses in general demonstrate a seasonal pattern of arriving in the fall and lasting through the winter months [47]. Based on epidemiological data on other human Coronavirus, it is likely that a second wave of COVID-19 infections will occur in fall 2020, though instead there could be a pattern of smaller peaks that recede when social distancing measures are implemented [48].

Given that it is unlikely that a vaccine will be widely available prior to 2021, a second wave of COVID-19 individuals in the fall of 2020 will pose unique challenges to public health and healthcare systems. First of all, this second wave would likely coincide with the onset of seasonal influenza. This would greatly challenge healthcare systems to respond to both diseases, including resulting in patient surges of in- fected individuals requiring hospitalization and intensive care and the need for increased medical equipment and supplies. Public health systems would also be challenged to respond, with a need to conduct surveillance for both diseases, promote and disseminate influenza vaccine, and conduct contact tracing. Intermittent use of social distancing, NPIs, and community restrictions may be required to control spread of both diseases and allow healthcare systems to manage the patient surges [6].

In the longer term, it is unknown what will happen with COVID-19. During past influenza pandemics, cases of the disease would trail off as mass vaccination was implemented and/or herd immunity levels were achieved. Because there is no long-term immunity to influenza, the pandemic influenza strain would then begin to join the annual influenza circulation cycle [6]. For example, the 2009 H1N1 influenza A strain continues to circulate and cause disease globally each year [6]. It is too early to tell if humans develop long-term immunity against COVID-19, but short term immunity is likely based on the fact that individuals infected with both SARS-CoV-1 and MERS CoV were found to have neutralizing antibodies for at least a few months after infection [49-51]. It is possible that SARS-CoV-2 could begin circulating annually along with other human Coronavirus. However, it is hopeful that an effective vaccine would be available before that happens.

\section{Other Emerging Public Health Concerns During the Pandemic}

As attention has turned to the public health response to contain the pandemic and support healthcare systems treating infected persons, direct and indirect public health implications are realized. From January 1-March 30, 2020, calls to poison centers in the US related to cleaning and disinfectant exposures increased by $20.4 \%$ when compared to the same time period in 2019 , and $16.4 \%$ compared to the same time period in 2018 [52]. These temporally-associated increases may correspond to increased use of these products to clean and disinfect surfaces for COVID-19 prevention, improper mixing of these products, not using appropriate PPE, using products incorrectly, and/or using them in poorly ventilated areas [52]. Recognizing the signs and symptoms of poisoning in patients seeking treatment and soliciting information about the ingested product(s) are paramount to early intervention and success of treatments. The pandemic has also been found to have led to a lower rate of routine pediatric vaccine administration, including measles vaccine, starting the week after COVID-19 was declared a national emergency [53]. The decline in measles-containing and other pediatric vaccines could lead to outbreaks of vaccine preventable diseases [53].

Stay-at-home orders and recommendations can lead to social isolation and increased loneliness, which are major risk factors for self-harm, anxiety, depression, and suicide [54]. In New York City, calls to one crisis intervention hotline increased $50 \%$ since the start of the pandemic; calls were related to changes in mood and anxiety, stressful life events, and interpersonal challenges $[55,56]$. Changes to psychological health could lead to increases in abuse of vulnerable persons, 
including domestic partners, children, and the elderly, as well as interpersonal violence [57]. More specifically, quarantine has been found to be associated with increases in negative psychological effects, such as symptoms of post-traumatic stress, fear, stigma, confusion, and anger [40], which could also lead to domestic abuse $[56,57]$. Nurses are mandated reporters when abuse or neglect are suspected and must therefore remain vigilant to the signs of abuse and neglect during this pandemic.

Interruptions in the healthcare system created by COVID-19-related surges can lead to delays in care for nonCOVID-19 conditions. As of May, 2020, the CDC recommends postponing preventive care, such as adult immunizations, lipid testing, and other screenings unless risks of postponement outweigh benefits. A number of professional organizations developed frameworks to aid in prioritizing care during periods of limited resource availability. For example, guidance developed by the American College of Surgeons [58] helps inform decision-making by surgeons and healthcare partners for procedures that can be delayed, alternatively treated, or scheduled and completed with considerations made to patient and personnel safety. The abrupt onset of the COVID-19 pandemic has seemingly taken precedence over the ongoing opioid crisis in the US, increasing the urgency to expand prescriptive privileges of buprenorphine to more providers to treat opioid use disorder [59].

The impact that interruptions like these will have on health outcomes remains to be seen, but diminished outcomes related to delays in care are of significant concern.

\section{Conclusion}

As of this writing (December, 2020), the SARS-CoV-2 which causes the disease COVID-19 has emerged into a global pandemic with no end in sight. This novel Coronavirus causes a highly contagious and deadly disease. It has led to healthcare surges that have exceeded hospital capacity in multiple communities and shortages of PPE, ventilators, and medical supplies across the U.S. Public health and healthcare have responded rapidly with non-pharmacological interventions that include surveillance, isolation, quarantine, contact tracing, and social distancing. Although COVID-19 vaccines now exist, mass vaccination and herd immunity from those vaccines are still months away. The U.S. will need to continue implementing social distancing measures and restrictions based on community infection rates until herd immunity is met. Nurses need to follow current protocols for patient care, including crisis standards of care.

\section{References}

1. Godderis L, Boone A, Bakusic J (2020) COVID-19: A new work-related disease threatening healthcare workers. Occup Med (Lond) 70: 315-316.

2. Holshue ML, DeBolt C, Lindquist S, et al. (2020) First case of 2019 novel coronavirus in the United States. N Engl J Med 382: 929936.

3. (2020) COVID-19 dashboard by the center for systems science and engineering. Johns Hopkins University.
4. Ji W, Wang W, Zhao X, et al. (2020) Cross-species transmission of the newly identified coronavirus 2019-nCoV. J Med Virol 92: 433-440.

5. Arora $\mathrm{P}$, Jafferany $\mathrm{M}$, Lotti $\mathrm{T}$, et al. (2020) Learning from history: Coronavirus outbreaks in the past. Dermatol Ther 2020: e13343.

6. Kissler SM, Tedijanto C, Goldstein E, et al. (2020) Projecting the transmission dynamics of SARS-CoV-2 through the post pandemic period. Science 368: 860-868.

7. Lau SKP, Luk HKH, Wong ACP, et al. (2020) Possible bat origin of severe acute respiratory syndrome coronavirus 2. Emerg Infect Dis 26: 1542-1547.

8. Ma J (2020) Coronavirus: China's first confirmed Covid-19 case traced back to November 17. South China Morning Post.

9. Ferioli M, Cisternino C, Leo V, et al. (2020) Protecting healthcare workers from SARS-CoV-2 infection: Practical indications. Eur Respir Rev 29: 200068.

10. Riou J, Althaus CL (2020) Pattern of early human-to-human transmission of Wuhan 2019 novel coronavirus (2019-nCoV), December 2019 to January 2020. Euro Surveill 25: 2000058.

11. Gandhi M, Yokoe DS, Havlir DV (2020) A symptomatic transmission, the Achilles' Heel of current strategies to control Covid-19. N Engl J Med 382: 2158-2160.

12. Arons MM, Hatfield KM, Reddy SC, et al. (2020) Presymptomatic SARS-CoV-2 infections and transmission in a skilled nursing facility. N Engl J Med 382: 2081-2090.

13. Rothan HA, Byrareddy SN (2020) The epidemiology and pathogenesis of coronavirus disease (COVID-19) outbreak. J Autoimmun 109: 102433.

14. World Health Organization (2020) Coronavirus disease 2019 (COVID-19) Situation Report - 96.

15. Wu Z, McGoogan JM (2020) Characteristics of and important lessons from the Coronavirus disease 2019 (COVID-19) outbreak in China: Summary of a report of 72314 cases from the chinese center for disease control and prevention. JAMA 323: 1239-1242.

16. Wilson N, Kvalsvig A, Barnard LT, et al. (2020) Case-fatality risk estimates for COVID-19 calculated by using a lag time for fatality. Emerg Infect Dis 26: 1339-1441.

17. Dong $\mathrm{Y}, \mathrm{Mo} \mathrm{X}, \mathrm{Hu} \mathrm{Y}$, et al. (2020) Epidemiological characteristics of 2143 pediatric patients with 2019 Coronavirus disease in China. Pediatrics e20200702.

18. Walker DM, Tolentino VR (2020) COVID-19: The impact on pediatric emergency care. Pediatr Emerg Med Pract 17: 1-27.

19. Schuchat A (2020) Public health response to the initiation and spread of pandemic COVID-19 in the United States, February 24-April 21, 2020. Morbidity and Mortality Weekly Report 69: 551-556.

20. Moriarty LF, Plucinski MM, Marston BJ, et al. (2020) Public health responses to COVID-19 outbreaks on cruise ships - worldwide, February-March 2020. MMWR Morb Mortal Wkly Rep 69: 347-352.

21. McMichael TM, Clark S, Pogosjans S, et al. (2020) COVID-19 in a long-term care facility - king county, Washington, February 27-March 9, 2020. MMWR Morb Mortal Wkly Rep 69: 339-342.

22. Kimball A, Hatfield KM, Arons M, et al. (2020) Asymptomatic and Presymptomatic SARS-CoV-2 infections in residents of a long-term care skilled nursing facility - king county, Washington, March 2020. MMWR Morb Mortal Wkly Rep 69: 377-381. 
23. Prem K, Liu Y, Russell TW, et al. (2020) The effect of control strategies to reduce social mixing on outcomes of the COVID-19 epidemic in Wuhan, China: A modelling study. Lancet Public Health 5: E261-E270.

24. McGrail DJ, Dai J, McAndrews KM, et al. (2020) Enacting national social distancing policies corresponds with dramatic reduction in COVID19 infection rates. PLoS One 15: e0236619.

25. Yusef D, Hayajneh W, Awad S, et al. (2020) Large outbreak of coronavirus disease among wedding attendees, Jordan. Emerg Infect Dis 26: 2165-2167.

26. Gaur S, Dumyati G, Nace DA, et al. (2020) Unprecedented solutions for extraordinary times: Helping long-term care settings deal with the COVID-19 pandemic. Infect Control Hosp Epidemiol 41: 729-730.

27. Esmailian M, Salehnia MH, Shirani M, et al. (2018) Reverse triage to increase the hospital surge capacity in disaster response. Adv J Emerg Med 2: e17.

28. (2020) Governor Cuomo holds a briefing on New York's COVID-19 response.

29. (2020) National survey shows dire shortages of PPE, hand sanitizer across the U.S.

30. Rebmann T, McPhee K, Haas GA, et al. (2018) Findings from an assessment and inventory of a regional, decentralized stockpile. Health Secur 16: 119-126.

31. Rebmann T, McPhee K, Osborne L, et al. (2017) Best practices for healthcare facility and regional stockpile maintenance and sustainment: A literature review. Health Secur 15: 409-417.

32. (2020) Coronavirus leaves federal stockpile of protective equipment nearly depleted. Associated Press.

33. Sweet L (2020) Trump said Pritzker has 'not performed well,' sends Illinois more ventilators after governor rips him over COVID-19 supplies. Chicago Sun Times.

34. Heinzerling A, Stuckey MJ, Scheuer T, et al. (2020) Transmission of COVID-19 to health care personnel during exposures to a hospitalized patient - Solano county, California, February 2020. MMWR Morb Mortal Wkly Rep 69: 472-476.

35. Centers for disease control and prevention (2020) Strategies to optimize the supply of PPE and equipment.

36. Chiu A (2020) Coronavirus-wracked nursing home evacuated after most of staff failed to show for two days. Washington Post.

37. Gold JA (2020) COVID-19: Adverse mental health outcomes for healthcare workers. BMJ 369: m1815.

38. Lai J, Ma S, Wang Y, et al. (2020) Factors associated with mental health outcomes among health care workers exposed to coronavirus disease 2019. JAMA Netw Open 3: e203976.

39. Du J, Dong L, Wang T, et al. (2020) Psychological symptoms among frontline healthcare workers during COVID-19 outbreak in Wuhan. Gen Hosp Psychiatry 67: 144-145.

40. Brooks SK, Webster RK, Smith LE, et al. (2020) The psychological impact of quarantine and how to reduce it: Rapid review of the evidence. Lancet 395: 912-920.

41. Kisely S, Warren N, McMahon L, et al. (2020) Occurrence, prevention, and management of the psychological effects of emerging virus outbreaks on healthcare workers: Rapid review and meta-analysis. BMJ 369: m1642.

42. Rebmann T (2020) Infectious disease disasters: Bioterrorism, emerging infections, and pandemics. In: Grota P, APIC Text of infection control and epidemiology ( $4^{\text {th }}$ edn), Association of Professionals in Infection Control and Epidemiology, Washington, DC, USA.

43. Haltiwanger J (2020) The US's top virus expert lowered expectations on a coronavirus vaccine after Trump's health secretary boasted about its development. Business Insider.

44. Rebmann T, Loux TM, Swick Z, et al. (2015) Are US jurisdictions prepared to dispense medical countermeasures through open points of dispensing? Findings from a national study. Health Secur 13: 96-105.

45. Rebmann T, Anthony J, Loux TM, et al. (2017) Assessment of closed point-of-dispensing (POD) preparedness in St. Louis County, Missouri, 2012-2016. Health Secur 15: 39-547.

46. (2020) Centers for disease control \& prevention. 1918 pandemic influenza: Three waves.

47. Killerby ME, Biggs HM, Haynes A, et al. (2018) Human coronavirus circulation in the United States 2014-2017. J Clin Viro 101: 52-56.

48. Moore KA, Lipsitch, M, Barry, et al. (2020) COVID-19: The CIDRAP viewpoint.

49. Al-Abdallat MM, Payne DC, Alqasrawi S, et al. (2014) Hospital-associated outbreak of middle east respiratory syndrome coronavirus: A serologic, epidemiologic, and clinical description. Clin Infect Dis 59: 1225-1233.

50. Liu W, Fontanet A, Zhang PH, et al. (2006) Two-year prospective study of the humoral immune response of patients with severe acute respiratory syndrome. J Infect Dis 193: 792-795.

51. Nie Y, Wang G, Shi X, et al. (2004) Neutralizing antibodies in patients with severe acute respiratory syndrome-associated coronavirus infection. J Infect Dis 190: 1119-1126.

52. Chang A, Schnall AH, Law R, et al. (2020) Cleaning and disinfectant chemical exposures and temporal associations with COVID-19 - national poison data system, United States, January 1, 2020-March 31, 2020. MMWR Morb Mortal Wkly Rep 69: 496498.

53. Santoli JM, Lindley MC, DeSilva MB, et al. (2020) Effects of the COVID-19 pandemic on routine pediatric vaccine ordering and administration. Morbidity and Mortality Weekly Report 69: 591593.

54. Holmes EA, O'Connor RC, Perry VH, et al. (2020) Multidisciplinary research priorities for the COVID-19 pandemic: a call for action for mental health science. Lancet Psychiatry 7: 547-560.

55. Williams K, Draper J, Clarke K (2020) Crisis hotlines are key to transforming care delivery. American Psychological Association Spring Highlights Meeting 2020

56. Brooks M (2020) COVID-19: Calls to NYC crisis hotline soar. Med Scape.

57. Tang K, Gaoshan J, Ahonsi B (2020) Sexual and reproductive health (SRH): A key issue in the emergency response to the coronavirus disease (COVID-19) outbreak. Reproductive Health 17: 59.

58. (2020) COVID-19: Elective case triage guidelines for surgical care. American College of Surgeons.

59. Green TC, Bratberg J, Finnell DS (2020) Opioid use disorder and the COVID 19 pandemic: A call to sustain regulatory easements and further expand access to treatment. Subst Abus 41: 147-149. 